\title{
บusisersily
}

\section{Preliminary Findings of a New Primary and Community Care Psychological Service in Northern Ireland: Low-Intensity Cognitive Behavioural Therapy for Common Mental Health Difficulties}

McDevitt-Petrovic, O., Kirby, K., McBride, O., Shevlin, M., Mc Ateer, D., Gorman, C., \& Murphy, J. (2018).

Preliminary Findings of a New Primary and Community Care Psychological Service in Northern Ireland: LowIntensity Cognitive Behavioural Therapy for Common Mental Health Difficulties. Behavioural and Cognitive Psychotherapy, 46(6), 761-767. https://doi.org/10.1017/S1352465818000322

Link to publication record in Ulster University Research Portal

Published in:

Behavioural and Cognitive Psychotherapy

Publication Status:

Published online: 13/06/2018

DOI:

$10.1017 / S 1352465818000322$

\section{Document Version}

Author Accepted version

\section{General rights}

Copyright for the publications made accessible via Ulster University's Research Portal is retained by the author(s) and / or other copyright owners and it is a condition of accessing these publications that users recognise and abide by the legal requirements associated with these rights.

\section{Take down policy}

The Research Portal is Ulster University's institutional repository that provides access to Ulster's research outputs. Every effort has been made to ensure that content in the Research Portal does not infringe any person's rights, or applicable UK laws. If you discover content in the Research Portal that you believe breaches copyright or violates any law, please contact pure-support@ulster.ac.uk. 


\title{
Preliminary Findings of a New Primary and Community Care Psychological Service in Northern Ireland: Low-Intensity Cognitive Behavioural Therapy for Common Mental Health Difficulties
}

Orla McDevitt-Petrovic, Karen Kirby*, Orla McBride, Mark Shevlin, Donal McAteer, Colin Gorman and Jamie Murphy

\begin{abstract}
Background: The prevalence of mental health difficulties in Northern Ireland (NI) in 2014/15 was $19 \%$, and this is $25 \%$ higher than in England. In recent years, there have been extensive consultations, and subsequent recommendations made in $\mathrm{NI}$, in an effort to address this. Aims: This study aims to evaluate the effectiveness of implementing an 'Improving Access to Psychological Therapies' (IAPT) stepped care service model using low intensity cognitive behavioural therapy (LI-CBT) in primary and community care settings in $\mathrm{NI}(\mathrm{NI})$ between 2014 and 2016. Method: A pilot intervention trial design utilised two standardised outcome measures (PHQ-9 and GAD-7) before treatment (at baseline), during treatment (in every session) and at discharge (at final session) ( $\mathrm{N}=199)$. Results: Preliminary reliable change outcomes for the pilot cohorts showed recovery rates of $47.9 \%$, improvement rates of $76.7 \%$ and deterioration rates of $6 \%$. Conclusions: These findings indicate that the IAPT service model is clinically effective in a NI population. Future analyses will also aim to identify individual and service level factors which potentially impact the effectiveness of the intervention.
\end{abstract}

Keywords: Effectiveness, Improving Access to Psychological Therapies (IAPT), Low intensity, Psychological wellbeing practitioners, Stepped Care. 
Initial evaluation of N.Ireland PWP service

\section{Introduction}

Improving Access to Psychological Therapies (IAPT) is a large scale initiative which has received substantial government investment in England (Gyani, Shafran, Layard \& Clark 2013). IAPT was first implemented in 2007 , and aims to improve access to evidenced-based psychological treatments for common mental health difficulties, primarily depression and anxiety (Clark et al., 2009). 'Access' in this case is considered to encompass a number of subconcepts, specifically availability, utilization, effectiveness, equity, efficiency and patient centeredness (Gulliford, Hughes \& Figeroa-Munoz, 2001).

The IAPT service model is informed by the National Institute for Health and Care Excellence (NICE) guidelines, which advocate the use of cognitive behavioural therapy (CBT) in the treatment of anxiety and depression (NICE 2004a, 2004b). Importantly, these guidelines also recommend that psychological interventions are delivered according to a stepped care framework, whereby the most effective, yet least resource-intensive, treatment is delivered first. NICE guidelines recommend that mild to moderate depression and anxiety can be managed effectively using low intensity interventions within primary care and community level settings (DHSSPS, 2005).

\section{Low Intensity Interventions}

Many low intensity or self-help interventions are framed theoretically in CBT and may be accessed with minimal or no practitioner contact or in tandem with therapeutic sessions (guided self-help). Low intensity in this case refers to forms of CBT guided self-help which can be delivered in non-traditional formats (e.g. via telephone/online) and often require less 
Initial evaluation of N.Ireland PWP service

practitioner support in terms of the frequency and duration of sessions. The IAPT workforce delivering these interventions are referred to as psychological wellbeing practitioners (PWPs) (Richards \& Whyte, 2011).

Evidence for the effectiveness of low-intensity interventions for depression and anxiety

Several studies and reviews have contributed to a robust evidence base for the effectiveness of CBT self-help materials in the treatment of depression and anxiety. Gellatly et al. (2007) conducted a meta-analysis on self- help and depression from which a large effect size was reported following guided self-help. The effect size was increased further in clients from non-clinical populations i.e. those who self- referred. Additional reviews have found evidence supporting the use of guided self-help with a cognitive behavioural framework in the treatment of anxiety disorders (Lewis, Pearce \& Bisson, 2012; Landreville, Gosselin, Grenier, Hodson \& Lorrain, 2016). Similarly Coull and Morris (2011) found guided self -help to be effective in the treatment of both depression and anxiety disorders, and again a greater effect with clients who had self-referred.

Systematic reviews and meta-analyses assessing behavioural activation have found it to be as effective as CBT and superior to counselling and brief psychodynamic therapy for depression (Cuijpers, van Starten \& Warmerdam, 2007; Ekers et al., 2008; Ekers et al., 2014). Furthermore behavioural activation has also been shown to be effective when received as a brief intervention (Hopko et al., 2003, Richards et al., 2016); it is considered to be particularly suitable for delivery as a low intensity intervention given that it is fairly uncomplicated, and does not necessitate complex skills from practitioners or clients (Cuijpers et al., 2007; Ekers et al., 2008). 
Initial evaluation of N.Ireland PWP service

\section{Effectiveness of the IAPT initiative}

IAPT services are routinely evaluated whereby a record of care is kept for each service user, detailing their progress throughout the course of treatment. A session by session monitoring protocol is used, ensuring that each service user has a clinical endpoint even where treatment is discontinued before expected. Details on the outcome measures used and the calculation of reliable change within IAPT services are provided within the method section of this extended report.

Evidence from UK IAPT sites suggests that the IAPT model is clinically effective, and more specifically that low intensity interventions are an effective treatment for mild to moderate depression and anxiety (Clark et al, 2009). Recovery rates increased steadily from $17 \%$ to $45 \%$ throughout the first three years in keeping with predictions that followed the pilot sites (Clark, 2011). The Reliable Change Index (Jacobson \& Truax, 1991) is used to determine reliable improvement and recovery rates within IAPT services, and this is described in more detail within the method section of this report. Gyani et al. (2013) examined year one data from 32 IAPT sites, representing approximately 19,000 clinical cases, and reported that $40 \%$ of individuals had reliably recovered and $64 \%$ had reliably improved by the end of treatment. In relation to low intensity interventions specifically, Gyani et al., (2013) concluded that guided self-help was a significant predictor of reliable recovery for those adults with a diagnosis of depression, general anxiety or comorbid depression and anxiety. Moreover the percentage of clients who were stepped up to a higher intensity service following guided self-help was 25.7 , compared to a figure of 54.5 for those who received pure self-help which lends further support to the superior efficacy of guided self-help. These findings were in keeping with NICE recommendations whereby guided self-help but not exclusive self-help is advocated for depression. Recent research using a sample of patients treated by a PWP delivering low intensity interventions across the first three years of IAPT, found that more than two thirds had reliably improved depression and anxiety symptoms by the end of treatment (Branson, Myles, Mahdi \& Shafran, 2018).

By 2013, IAPT clinical services were treating approximately 400,000 individuals annually; almost half of these had reliably recovered by the end of treatment (Layard and Clark 2015). 
Initial evaluation of N.Ireland PWP service

The most recently published figures indicate that $51 \%$ of eligible referrals within IAPT services, achieved recovery criteria, with rates therefore remaining above the national target of $50 \%$ (HSC, 2017).

Primary Care Level Psychological Therapies in Northern Ireland

The prevalence of general mental health problems in Northern Ireland (NI) was $19 \%$ in 2014/15, and this is 25\% higher than in England (DHSSPS, 2014). Despite this, services in England spend more than double the per capita spend on the provision of support for individuals with mental health difficulties (DHSSPS, 2010; DHSSPS, 2014).

Although the efficacy of early interventions such as low intensity cognitive behavioural therapy (LI-CBT) is most strongly evidenced with depression and anxiety (Gellatly et al., 2007; Ekers et al., 2014), and although such approaches are shown to be cost-effective in the long term (Layard et al., 2007), a stepped care model has not yet been fully established in NI (Blane, Williams, Morrison, Wilson \& Mercer, 2014).

Psychological therapy service provision in NI has tended to focus on the more complex end of the spectrum of mental health difficulties, and consequently, educational and professional training has similarly concentrated largely on individuals working at these levels (HSC, 2014). In 2013, the Northern Ireland Mental Health Services Threshold Criteria acknowledged that low intensity therapists working at stepped care levels one and two, including those delivering low intensity interventions are crucial for the establishment of an effective stepped care approach to psychological therapy provision in the province (HSC, 2014).

\section{Training of Psychological Wellbeing Practitioners in Northern Ireland}

In light of the mental health problems faced by large numbers of the population in Northern Ireland, and in recognition of the need for an appropriately skilled and experienced workforce to fill the aforementioned gap in service provision, the content of the MSc applied psychology course at Ulster University was modified in 2014. This has since facilitated training in evidence-based low intensity interventions for common mental health 
Initial evaluation of N.Ireland PWP service

difficulties. These trainee PWPs (accredited by the British Psychological Society), deliver low intensity cognitive behavioural therapy interventions to individuals within

Primary/Community Care level services throughout training. The IAPT training curriculum for PWPs includes compulsory training in the use of CBT- based self-help materials (Richards and Whyte, 2011). Trainee PWPs attend university two days per week, and have one directed learning day on which a guided task is completed independently (e,g directed reading with written exercise).

Two days are also spent seeing clients at the designated placement site. Trainees also attend mandatory individual clinical case management on a weekly basis, and group clinical skills supervision sessions on a fortnightly basis as per IAPT accreditation requirements; all supervisors are BABCP accredited practitioners and completed the IAPT PWP supervision training course (Richards and Whyte, 2011).

\section{Aims and Objectives}

The current provision of psychological therapies at the Primary/Community Care Level in NI is in its early stages of development, with few empirical evaluations to date of the effectiveness of treatment approaches at this level. Indeed, recent reports indicated that access to psychological services has been wholly inadequate and significant funding is required to match government spending in England (DoH, 2016; Betts \& Thompson, 2017). Hence, there is a need to provide evidence of a service model that works, and can be implemented effectively in NI. The IAPT initiative is not formally implemented in Northern Ireland as in England. Elements of the IAPT clinical model have been applied for the purposes of the current study. More specifically, the main objective of the current study is to present the preliminary findings of an evaluation of PWPs providing low intensity interventions for common mental health difficulties working directly with three of the newly 
Initial evaluation of N.Ireland PWP service

established 'Primary care Talking Therapies Hubs', other primary care (GP practices) and community care settings in NI. Specifically, the preliminary study predicted that following a course of LI-CBT, there would be a reduction in PHQ-9 and GAD-7 scores demonstrative of reliable improvement and/or recovery, and that these findings would be similar to existing IAPT UK outcomes, thereby providing initial evidence that this element of the IAPT service model is effective in an NI context.

\section{Methods}

Design

This study presents findings from the first wave of a larger ongoing longitudinal study design, using pre-post interventional trial methods, following clients $(\mathrm{N}=199)$ from baseline (before commencement of therapy), through the course of low intensity weekly treatment (1-11) sessions, and at discharge (final session of treatment). The current preliminary study is limited to examining changes in psychological status in clients before and after therapy.

\section{Measures}

As per IAPT service protocol, each client completed two routine outcome measures at every session. The Patient Health Questionnaire (PHQ-9) (Kroneke, Spitzer \& Williams, 2001), was used to measure the severity of depressive symptoms. This is a nine-item standardised measure which has been validated in a UK depressed population (Cameron, Crawford, Lawton \& Reid, 2008). The scores range from 0 to 27 , with a score of ten or more being the threshold to identify clinically relevant depressive symptoms (Kroneke, Spitzer \& Williams, 2001).

The General Anxiety Disorder Questionnaire (GAD-7) (Spitzer, Kroneke, Williams \& Lowe, 2006), has also been determined to have good psychometric properties having been validated in U.S. populations. The scores range from 0 to 21 with a score of eight or more 
Initial evaluation of N.Ireland PWP service

being the threshold to identify clinically relevant general anxiety disorder, and it has adequate sensitivity and specificity to screen for post-traumatic stress, panic disorder or social anxiety disorder (Kroneke, Spitzer, Williams, Monahan \& Lowe, 2007).

Caseness and reliable change calculations

In keeping with IAPT recommendations (Gyani et al., 2013), the clinical outcomes reported here are related only to clients meeting "caseness" criteria. This required at least two contacts with a PWP, as pre and post treatment scores cannot be collected based on a single initial session. A 'case' client must also have scored above the clinical thresholds on at least one of the measures at assessment; more specifically, this refers to scores of 10 or above on the PHQ-9 and/or 8 or above on the GAD-7. 163 clients were identified as "case" and 34 were identified as "non-case" prior to analysis, in accordance with the IAPT "caseness" criteria.

The initial calculations of 'recovery' within IAPT services, revealed a number of issues with the process, namely that it did not account for psychometric measurement errors, and the binary characteristic also disregarded improvements which may have occurred in the absence of recovery. Furthermore it provided no details in relation to clinical deterioration. Consequently the current study has used a modified reliable recovery index as recommended by Gyani et al., (2013). The Reliable Change Index (Jacobson \& Truax, 1991) is an appropriate way of assessing deterioration or improvement in anxiety and depression 
Initial evaluation of N.Ireland PWP service

symptoms, as it allows one to determine whether an increase or decrease in measure scores from baseline to post-treatment exceeds the measurement error of the relevant scale, and thereby can be considered statistically reliable. In keeping with existing IAPT evaluations (Gyani et al., 2013), a reduction or increase of six or more points on the PHQ-9 and a reduction or increase of four or more points on the GAD-7 have been determined as the thresholds for reliable change in depression and anxiety symptoms respectively (see figure 2). Clients are considered to have 'reliably improved' if either of their measure scores reliably decreased and the score for the other measure either remained the same or did not reliably deteriorate. Clients are considered to have 'reliably deteriorated' if either measure score reliably increased, or the other score either also increased or did not reliably improve.

A reliable recovery index was also used in line with existing IAPT studies (Gyani et al., 2013). Clients are considered to be 'reliably recovered' if they scored above the clinical threshold on at least one of the outcome measures at assessment interview, showed reliable improvement during the course of treatment, and scored below clinical thresholds on both the PHQ-9 and GAD-7 at the point of treatment completion.

\section{Interventions}

Trainee PWPs had successfully completed modules one and two of the curriculum at this point of commencing clinical placement, specifically those relating to assessment and intervention (Richards and Whyte, 2009). All trainee PWPs had similar educational backgrounds given that all had completed an undergraduate psychology degree at the time of training, and all were enrolled on the MSc applied psychology course (mental health and psychological therapies). In keeping with the IAPT service model, PWP's (N=29), were required to support clients to implement a range of low-intensity treatments. All trainee PWPs at initial contact with a client, completed a standardised assessment interview, the purpose of which was also to determine a client's suitability for low intensity treatment. During assessment the PWP collected baseline outcome measure scores, and determined the nature and severity of symptoms, level of risk, and the patient's motivation and capability to participate in the intervention. Treatment goals and plans were discussed after 
Initial evaluation of N.Ireland PWP service

collaborative formulation of a problem statement. Exclusion criteria at the assessment included, significant risk of harm to self or others, ongoing substance abuse and severe or enduring mental illness. The low intensity clinical procedures implemented by PWP's include behavioural activation, cognitive restructuring, exposure therapy, problem solving, managing panic, sleep hygiene, and medication support. Although this is not an exhaustive list of interventions, it does represent the core clinical procedures that PWP's use with their clients (Richards \& Whyte, 2011). In keeping with the stepped care framework (Scogin, Hanson \& Welsh, 2003), the systematic monitoring of client's therapeutic progress by the trainee PWPs and their clinical supervisors ensured clients were stepped up to a higher intensity service where it was deemed to be clinically required i.e where there had been an inadequate response to low intensity treatments ( Seekles,van Straten, Beekman, Marwijk and Cuijpers, 2011).

\section{Sample}

Data from a total of 199 clients who were referred to a trainee PWP between January 2015 and June 2015, and between January 2016 and June 2016 were collected. Referrals were received from sources which included GPs, Primary Care Team, Talking Therapies Hub, and the client (self-referral). 198 of these referrals subsequently completed an assessment with a trainee PWP, and 1 referral did not, as exclusion criteria were apparent within the referral letter. 178 of the clients who completed assessment attended at least a second contact. A number of clients did not attend a second session due to either non-attendance or being stepped up to a higher intensity service ( $N=20$. The rate of uptake of LICBT was $89.4 \%$. The duration of treatment for case clients ranged between 2 and 11 sessions, with a mean of 5.7 sessions.

Caseness criteria was achieved by 163 referrals of which there were 104 females and 59 males. Ages ranged from 18 to 77 , with a mean age of $39(S D=13.56)$ (see Figure 1 ). A total of 113 case clients were discharged following successful treatment completion, and 50 were discharged before treatment completion due to either non-attendance or step up. Community settings within which a variety of wellbeing services are offered, facilitated treatment for $55 \%$ of case clients, $18 \%$ were treated in a GP practice, and $27 \%$ were treated 
Initial evaluation of N.Ireland PWP service

at primary care psychological therapies service within a NI NHS Trust as per a stepped care framework. In relation to initial presentations, $25 \%$ of case clients demonstrated depressive symptoms, $14 \%$ presented with general anxiety and $47 \%$ with comorbid depression and anxiety. The primary presentations of the remaining clients included specific phobia, panic disorder (with or without agoraphobia), health anxiety, and obsessive compulsive symptoms.

Figure 1 about here

\section{Ethics statement}

Full ethical approval was obtained from ORECNI, with additional clinical governance obtained from three Trust sites in NI.

IRAS ref 181559/ REC ref 16/LO/0343.

\section{Results}

In relation to depression $59.5 \%$ of clients achieved reliable improvement and $3 \%$ showed reliable deterioration. In relation to anxiety $70.6 \%$ of clients achieved reliable improvement and $5.5 \%$ showed reliable deterioration. Overall, $47.9 \%$ of clients who met caseness criteria 
Initial evaluation of N.Ireland PWP service

demonstrated reliable recovery, $76.7 \%$ of these clients achieved reliable improvement, and $6.1 \%$ demonstrated reliable deterioration (see table 1). There was no proportional difference observed in overall reliable recovery rates between clients treated in Northern Ireland and those treated in England at the same time period $\left[x^{2}(1, N=163)=0.17, p>0.5\right]$. However, the proportion of clients treated in Northern Ireland who demonstrated overall reliable improvement was significantly higher when compared with rates in England, $\left[\chi^{2}(1\right.$, $N=163)=14.57, p<0.001]$.

\section{Table 1 about here}

\section{Discussion}

Initial Northern Ireland outcomes: Clinical effectiveness

Findings from the current study are in keeping with outcomes from UK IAPT sites including the previously reported 2015/16 outcomes (HSC, 2016). More specifically, when NI reliable change rates are compared directly with these, recovery rates are $47.9 \%$ and $46.3 \%$ respectively. Improvement rates are $76.7 \%$ and $62.2 \%$ for $\mathrm{NI}$ and England respectively (HSC, 2016). These preliminary results provide initial evidence that low intensity cognitive behavioural therapy is an effective treatment for mild to moderate level mental health difficulties in NI.

IAPT outcomes consistently report higher improvement rates compared to recovery rates, given that improvement considers only the scale of change, and not whether the client has moved below clinical caseness. It is important to acknowledge that the service evaluated in the current report is not an official IAPT service, and trainee PWPs were not working under the usual high volume caseload. This may partly account for the higher improvement rates 
Initial evaluation of N.Ireland PWP service

presented for Northern Ireland; the sample in the current evaluation is considerably smaller than numbers treated by IAPT services in England. Furthermore, the overall outcomes reported by UK IAPT services, consider those who may have been treated by both low and high intensity therapies throughout the course of treatment. Importantly, reports on the use of IAPT services highlight the fact that a patient's therapy type can change between appointments. Therefore, two or more therapy types may be used within a single referral, meaning a client may have received both low and high intensity interventions. Although the current report includes the outcomes of those who were stepped up to higher intensity care, only low intensity interventions were delivered and evaluated. Consequently, a higher proportion of suitable referrals within the Northern Ireland sample, may partly account for the higher improvement rates reported here.

As reported earlier the prevalence of mental difficulties in $\mathrm{NI}$ is $25 \%$ higher than in England (DHSSPS, 2014). More explicitly, the Northern Ireland Health Survey (2014/15) reported that $19 \%$ of respondents exhibited symptoms of a potential mental health problem (Bell \& Scarlett, 2015). It would be reasonable to assume that at least some of this population would be suitable for low intensity interventions, and that an established PWP service in Northern Ireland may contribute to reducing the prevalence of difficulties by improving access to psychological therapies at the primary care level, as the IAPT initiative has done in England. (Layard and Clark, 2015).

It is also important to acknowledge that LICBT interventions in the current study were delivered by trainee PWPs. Recent research has indicated clients treated by qualified PWPs had superior clinical outcomes when compared with trainee PWPs, although further research investigating relationships between therapist experience and competence, and patient outcomes is needed, particularly in relation to low intensity interventions (Branson, Myles, Mahdi \& Shafran, 2017).

Adhering to the evidence base: Delivering the right treatment, at the right time, in the right place

As outlined earlier, the IAPT service model is informed by evidence based clinical guidelines. Our findings support these NICE guidelines in a NI context, which advocate the use of 
Initial evaluation of N.Ireland PWP service

cognitive behavioural therapy for depression and anxiety. NICE assemble a panel of experts comprising clinicians, researchers and consumers who carefully review the available evidence base on the optimum treatments for each physical and mental health problem. In 2004, NICE carried out systematic reviews of research investigating the effectiveness of interventions for depression and anxiety disorders. The resultant clinical guidelines advocate the provision of specific kinds of cognitive behavioural therapy (CBT) for depression and anxiety disorders (NICE, 2004). Crucially, cognitive behavioural therapy is considered to be more effective than medication given that it reduces the likelihood of relapse by at least $50 \%$, and moreover, the vast majority of clients prefer it to a pharmacological treatment (McHugh, Whitton, Peckham, Welge and Otto, 2013).

NICE guidelines further recommend that mild to moderate depression and anxiety can be managed effectively using low intensity interventions within primary care level settings (DSSPS, 2005). Furthermore, low intensity interventions improve the flexibility, capacity and responsiveness of the relevant services while increasing patient-choice, and enhancing service cost-effectiveness (Bennett-Levy et al., 2012).

\section{Limitations}

The absence of a control group is an acknowledged limitation of the current pilot study, however this will be included in future evaluations which will involve analysis of a larger sample and predictors of treatment outcome.

\section{Conclusion}

Currently, the mental health service framework in NI does not formally apply NICE evidence, which has also been highlighted in the recent Evaluation of the Bamford Action Plans (DoH, 2015). Our findings indicate that reliable recovery and improvement have been demonstrated for clients in NI who have presented with common mental health difficulties. 
Initial evaluation of N.Ireland PWP service

Indeed, LI-CBT interventions (provided by PWPs) provides the only evidence base to date in $\mathrm{NI}$ for treating anxiety and depression at stepped care levels 1-3. Additionally, the Bamford evaluation highlighted a need to further promote psychological therapies, to improve access to services in times of crisis, and to improve involvement at the community and voluntary level (DOH, 2016; Betts \&Thompson, 2017). Our initial findings indicate that PWP's could potentially address this need in light of promising clinical outcomes.

Recommendations and plans for future low intensity CBT research in Northern Ireland

\section{Clinical Policy Recommendations:}

The current pilot offers a potential solution to addressing the need for evidence-based treatment and suggests that low intensity CBT (provided by accredited PWPs), is a clinical and cost-effective intervention, as well as being an effective service model within primary and community care settings in NI (stepped care 1-3).Therefore, our recommendation to practice and policy implementation, is that in order to assist the people of $\mathrm{NI}$ to gain access to appropriate, clinical and cost effective psychological interventions at steps 1-3, a province-wide PWP service, embedded within the talking therapy hubs, and GP practices (a recent recommendations made by Sands, 2017) needs to be fully established and adequately funded. This could potentially prevent more complex mental health issues through the use of early intervention and quicker access to services with reduced waiting times, and by enabling service users to access the right treatment in the right place at the right time. PWP's are fit for purpose and fit for the future of $\mathrm{NI}$ mental health services.

\section{Research recommendations:}


Initial evaluation of N.Ireland PWP service

The collection of client data and routine outcome measures is continuing at pre-treatment, post treatment and follow up points. Future analyses will focus on this larger sample of all previous, current and future cohorts combined. Reliable recovery, improvement and deterioration rates will be produced. Previous IAPT studies have determined that many of the psychological benefits resulting from interventions had been maintained (Clark et al., 2009). In order to produce comparative data regarding longer-term effectiveness, our future research will also examine data collected from clients at a four month follow-up point after discharge from the service. In order to test for individual differences in treatment effect, a series of latent growth curve models will be specified and tested in order to determine if there are different rates of change for unobservable sub- populations, and to identify individual or service level variables which potentially increase or reduce the effectiveness of the intervention. Furthermore, data on employment statuses using the WSAS, and medication usage pre and post treatment will be scrutinised.

Increasingly, governments are driven by the recognition of the huge economic and social costs of high prevalence disorders, and our future research should also evaluate cost effectiveness in order to inform future service design and planning. Randomised controlled trials facilitating a comparison of service models may also provide further evidence for the effectiveness of the IAPT model in a Northern Ireland context.

\section{Role of the funding source}

Orla McDevitt-Petrovic is funded by a studentship granted by DEL. This research did not receive any specific grant from funding agencies in the public, commercial, or not-for-profit sectors.

\section{Conflict of interest}

The authors report no conflict of interest. 
Initial evaluation of N.Ireland PWP service

\section{Acknowledgments}

Ulster University would like to acknowledge the trainee PWPs and placement providers involved in delivering the intervention which has been evaluated. 
Initial evaluation of N.Ireland PWP service

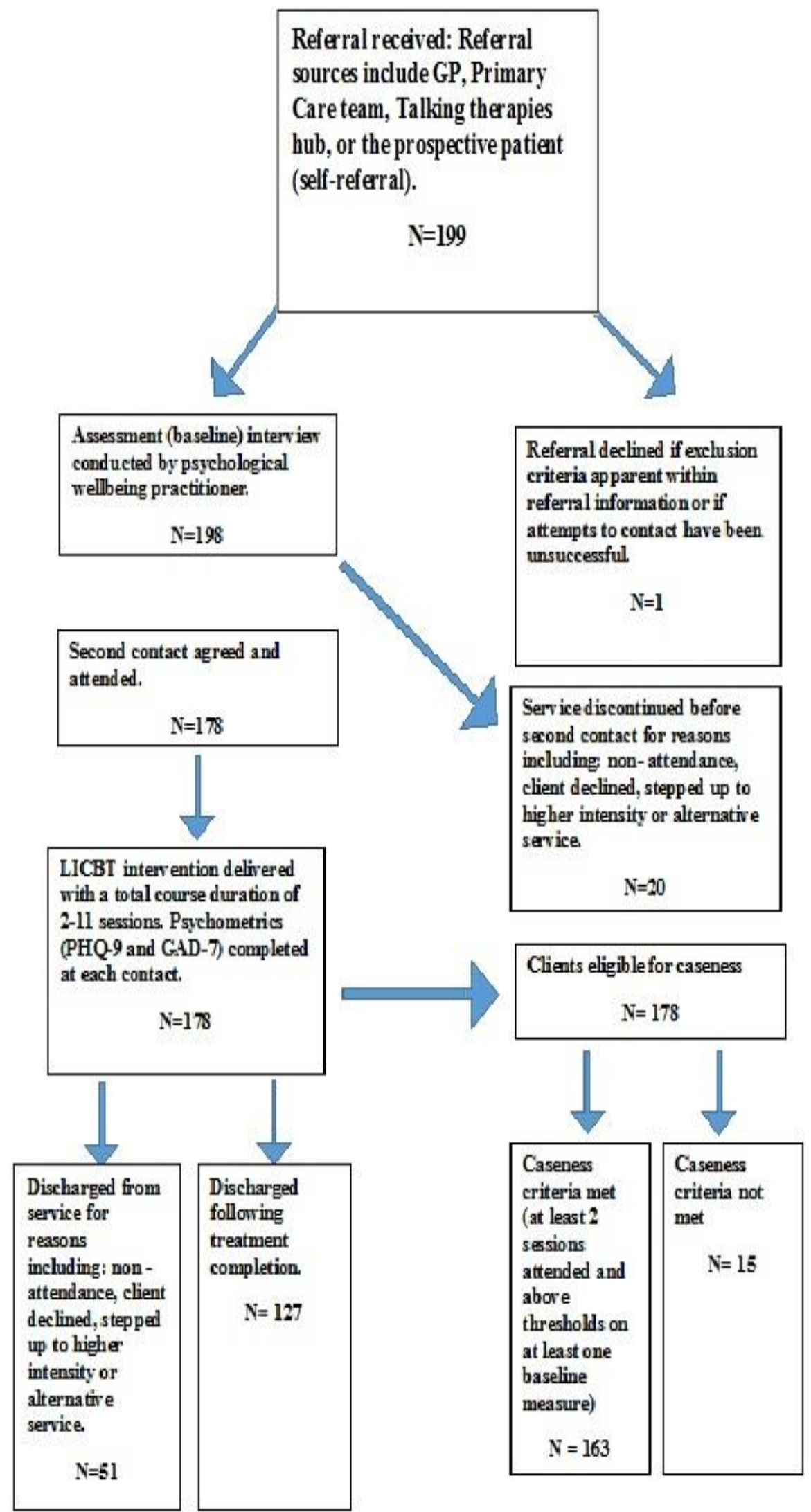

Figure 1: Pathways from referral to discharge points for patient cahorts 2015-2016 
Table 1: Frequencies and percentages of reliable recovery, improvement and deterioration rates for all clients meeting caseness criteria ( $\mathrm{n}=163$ ).

\begin{tabular}{|c|c|c|c|c|c|c|c|}
\hline & \multicolumn{2}{|c|}{ Reliable Improvement } & \multicolumn{2}{|c|}{ Reliable Deterioration } & \multirow{2}{*}{$\begin{array}{l}\text { Overall Reliable } \\
\text { Improvement }\end{array}$} & \multirow{2}{*}{$\begin{array}{l}\text { Overall Reliable } \\
\text { Deterioration }\end{array}$} & \multirow{2}{*}{$\begin{array}{c}\text { Overall Reliable } \\
\text { Recovery }\end{array}$} \\
\hline & (PHQ-9) & (GAD-7) & (PHQ-9) & (GAD-7) & & & \\
\hline Yes & $97(59.5 \%)$ & $115(70.6 \%)$ & $5(3.1 \%)$ & $9(5.5 \%)$ & $125(76.7 \%)$ & $10(6.1 \%)$ & $78(47.9 \%)$ \\
\hline No & $66(40.5 \%)$ & $48(29.4 \%)$ & 158 (96.9\%) & 154 (94.5\%) & $38(23.3 \%)$ & 153 (93.9\%) & 85 (52.1\%) \\
\hline
\end{tabular}

Notes: *At least 2 sessions attended and above clinical thresholds on one or both measures at baseline = caseness . 


\section{References}

Bell, C., \& Scarlett, M. (2015). Health Survey Northern Ireland: First Results 2014/15.

Bennett-Levy, J. E., Butler, G. E., Fennell, M. E., Hackman, A. E., Mueller, M. E., \& Westbrook, D. E. (2004). Oxford guide to behavioural experiments in cognitive therapy. Oxford University Press.

Betts, J and Thompson, J (2017). Mental Health in Northern Ireland: Overview, Strategies, Policies, Care Pathways, CAMHS and Barriers to Accessing Services. Research and Information Service Research Paper. Northern Ireland Assembly.

Blane, D. N., Williams, C., Morrison, J., Wilson, A., \& Mercer, S. (2014). Psychological therapies in primary care: a progress report.

Branson, A., Myles, P., Mahdi, M., \& Shafran, R. (2018). The Relationship between Competence and Patient Outcome with Low-Intensity Cognitive Behavioural Interventions. Behavioural and cognitive psychotherapy, 46(1), 101-114.

Cameron, I. M., Crawford, J. R., Lawton, K., \& Reid, I. C. (2008). Psychometric comparison of PHQ-9 and HADS for measuring depression severity in primary care. Br J Gen Pract, 58(546), 32-36.

Clark, D. M. (2011). Implementing NICE guidelines for the psychological treatment of depression and anxiety disorders: the IAPT experience. International Review of Psychiatry, 23(4), 318-327.

Clark, D. M., Layard, R., Smithies, R., Richards, D. A., Suckling, R., \& Wright, B. (2009). Improving access to psychological therapy: Initial evaluation of two UK demonstration sites. Behaviour research and therapy, 47(11), 910-920.

Coull, G., \& Morris, P. G. (2011). The clinical effectiveness of CBT-based guided self-help interventions for anxiety and depressive disorders: a systematic review. Psychological Medicine, 41(11), 2239-2252.

Cuijpers, P., Van Straten, A., \& Warmerdam, L. (2007). Behavioral activation treatments of depression: A meta-analysis. Clinical psychology review, 27(3), 318-326.

Department of Health and NHS (2014). Achieving Better Access to Mental Health Services by 2020.

Department of Health Social Sciences and Public Safety (2014). Bamford monitoring report.

Department of Health Social Sciences and Public Safety (2015) Delivering the Bamford vision: The Response of the Northern Ireland Executive to the Bamford review of Mental Health and Disability Action Plan 2012-2015.

Department of Health SS, AND Public Safety. IAPT outline specification: Improving Access To Psychological Therapies (IAPT) 2005. 
Initial evaluation of N.Ireland PWP service

Department of Health, Social Services and Public Safety. A strategy for the development of psychological therapies services. Northern Ireland: Department of Health Social Services and Public Safety (2010).

Department of Health, Social Services and Public Safety. Making Life Better: A whole system strategic framework for public health 2013-2023. Department of Health, social Services and Public Safety: Belfast. (2014).

Department of Health, Social Services and Public Safety. Reshaping the System: Implications for Norther Ireland's Health and Social Care Services of the 2010 Spending Review (McKinsey Report). Belfast: Belfast. (2010).

Department of Health, Social Services and Public Safety. Health and Wellbeing 2026 Delivering Together. (2016).

Ekers, D., Richards, D., \& Gilbody, S. (2008). A meta-analysis of randomized trials of behavioural treatment of depression. Psychological medicine, 38(5), 611-623.

Ekers, D., Webster, L., Van Straten, A., Cuijpers, P., Richards, D., \& Gilbody, S. (2014). Behavioural activation for depression; an update of meta-analysis of effectiveness and sub group analysis. PloS one, 9(6), e100100.

Gellatly, J., Bower, P., Hennessy, S., Richards, D., Gilbody, S., \& Lovell, K. (2007). What makes self-help interventions effective in the management of depressive symptoms? Metaanalysis and meta-regression. Psychological medicine, 37(9), 1217-1228.

Gulliford, M., Hughes, D., Figeroa-Munoz, J., \& Guy's, King's and St Thomas' School of Medicine, London (United Kingdom). Public Health and Health Services Research Group; (2001). Access to Health Care: Report of a Scoping Exercise for the National Co-ordinating Centre for NHS Service Delivery and Organisation R and D (NCCSDO) 26 February 2001 (with Minor Amendments August 2001). Guy's, King's and St Thomas' School of Medicine, Public Health and Health Services Research Group.

Gyani, A., Shafran, R., Layard, R., \& Clark, D. M. (2013). Enhancing recovery rates: lessons from year one of IAPT. Behaviour Research and Therapy, 51(9), 597-606.

Health and Social Care Board (2014) You In Mind. Mental Healthcare Pathway.

Health and Social Care Information Centre (2016), Psychological Therapies, Annual Report on the use of IPAT services.

Health and Social Care Information Centre (2017), Psychological Therapies, Annual Report on the use of IPAT services.

Hopko, D. R., Lejuez, C. W., Ruggiero, K. J., \& Eifert, G. H. (2003). Contemporary behavioral activation treatments for depression: Procedures, principles, and progress. Clinical psychology review, 23(5), 699-717.

Jacobson, N. S., \& Truax, P. (1991). Clinical significance: a statistical approach to defining meaningful change in psychotherapy research. Journal of consulting and clinical psychology, 59(1), 12. 
Initial evaluation of N.Ireland PWP service

Kroenke, K., Spitzer, R. L., \& Williams, J. B. (2001). The Phq-9. Journal of general internal medicine, 16(9), 606-613.

Kroenke, K., Spitzer, R. L., Williams, J. B., Monahan, P. O., \& Löwe, B. (2007). Anxiety disorders in primary care: prevalence, impairment, comorbidity, and detection. Annals of internal medicine, 146(5), 317-325.

Landreville, P., Gosselin, P., Grenier, S., Hudon, C., \& Lorrain, D. (2016). Guided self-help for generalized anxiety disorder in older adults. Aging \& mental health, 20(10), 10701083.

Layard, R., Clark, D., Knapp, M., \& Mayraz, G. (2007). Annex D: Cost-benefit analysis of psychological therapy. Department of health Improving Access to Psychological Therapies (IAPT) programme: An outline business case for the national rollout of local psychological therapy services. London: Department of Health.

Layard, R., \& Clark, D. M. (2015). Why more psychological therapy would cost nothing. Frontiers in psychology, 6, 1713.

Lewis, C., Pearce, J., \& Bisson, J. I. (2012). Efficacy, cost-effectiveness and acceptability of self-help interventions for anxiety disorders: systematic review. The British journal of psychiatry, 200(1), 15-21.

McHugh, R. K., Whitton, S. W., Peckham, A. D., Welge, J. A., \& Otto, M. W. (2013). Patient preference for psychological vs. pharmacological treatment of psychiatric disorders: a meta-analytic review. The Journal of clinical psychiatry, 74(6), 595.

National Institute for Clinical Excellence, \& Britain, G. (2004). Anxiety: management of anxiety (panic disorder, with or without agoraphobia, and generalised anxiety disorder) in adults in primary, secondary and community care. National Institute for Clinical Excellence.

National Collaborating Centre for Mental Health. (2004). Depression: management of depression in primary and secondary care. London: National Institute for Clinical Excellence.

Richards DA, Whyte M. Reach Out: National programme student materials to support the training and for Psychological Wellbeing Practitioners delivering low intensity interventions. UK: Rethink Mental Illness, 2011.

Richards, D. A., Ekers, D., McMillan, D., Taylor, R. S., Byford, S., Warren, F. C., ... \& O'Mahen, H. (2016). Cost and Outcome of Behavioural Activation versus Cognitive Behavioural Therapy for Depression (COBRA): a randomised, controlled, non-inferiority trial. The Lancet, 388(10047), 871-880.

Sands, Louise (2017). RCGP Associate Director - Policy Reform seminar on improving mental health provision in NI, 17th Jan 2017), as cited in Betts, J and Thompson, J (2017). Mental Health in Northern Ireland: Overview, Strategies, Policies, Care Pathways, CAMHS and Barriers to Accessing Services. Research and Information Service Research Paper. Northern Ireland Assembly.

Scogin, F. R., Hanson, A., \& Welsh, D. (2003). Self-administered treatment in stepped-care models of depression treatment. Journal of clinical psychology, 59(3), 341-349. 
Initial evaluation of N.Ireland PWP service

Seekles, W., van Straten, A., Beekman, A., van Marwijk, H., \& Cuijpers, P. (2011). Stepped care treatment for depression and anxiety in primary care. a randomized controlled trial. Trials, 12(1), 171.

Spitzer, R. L., Kroenke, K., Williams, J. B., \& Löwe, B. (2006). A brief measure for assessing generalized anxiety disorder: the GAD-7. Archives of internal medicine, 166(10), 1092-1097. 